\title{
Espiritualidade nos cuidados paliativos de pacientes oncológicos
}

\author{
Thayná Cristhina Soares Marques* (1) \\ Silvia Helena Modenesi Pucci (1) \\ Universidade de Santo Amaro, São Paulo, SP, Brasil
}

\begin{abstract}
Resumo: A espiritualidade como ferramenta aos cuidados paliativos de pacientes oncológicos vem sendo cada vez mais abordada no contexto acadêmico, como integrante da qualidade de vida. Este estudo analisa de que forma a espiritualidade pode influenciar positivamente no tratamento oncológico e em cuidados paliativos, e contribuir com a melhora da qualidade de vida destes pacientes. Foram selecionados 29 artigos nacionais que evidenciaram a espiritualidade como variável positiva no enfrentamento aos cuidados paliativos, tanto para paciente quanto familiares e equipe multidisciplinar. A espiritualidade é utilizada como ferramenta pela equipe de cuidados paliativos como auxiliadora na compreensão do impacto causado pelo diagnóstico e cuidado integral do paciente, possibilitando ao profissional psicólogo intervir sobre o paciente e sua rede. Ao paciente e sua família, a espiritualidade atua como estratégia de enfrentamento e conforto. Este estudo encontrou limitações acerca de pesquisas com pacientes infantis/adolescestes e sua família.
\end{abstract}

Palavras-chave: espiritualidade, cuidados paliativos, paciente oncológico, psicologia.

\section{Introdução}

A espiritualidade passou a ser vista pelo mundo acadêmico e pelos profissionais da saúde não só como termo místico, mas como uma das integrantes do espectro que compõe a qualidade de vida de um sujeito.

Ao contrário de religiosidade, a espiritualidade não está ligada a uma instituição ou a um segmento de doutrinas, mas a auxiliar o sujeito a embasar suas significações, seu contato com o transcendente e a forma com a qual ele processa suas vivências, ou seja, o modo de vida e adaptação do indivíduo. Por meio do reconhecimento dessa perspectiva da espiritualidade foi necessário enquadrá-la como integrante de qualidade de vida para o sujeito, principalmente aqueles em tratamento oncológico (Panzini, Rocha, Bandeira, \& Fleck, 2007).

O diagnóstico oncológico é acompanhado por um estigma que amedronta toda a sociedade, em especial aquele que o recebe. Isso se dá devido ao grande índice de mortalidade causado pela patologia. Mesmo com o aumento de métodos curativos e medicamentosos, ainda existem os casos em que não há remissão da doença, passando a ser possível, a este paciente, somente os cuidados paliativos (Elias, 2003).

Os cuidados paliativos se mostram, ao paciente em contato com a finitude de sua vida, de modo diferente dos demais cuidados que lhe foram oferecidos, pois este não tem intenção curativa, mas proporcionar ao paciente, qualidade de vida em seus últimos dias. Para isso, existe uma equipe multiprofissional para tentar proporcionar que

*Endereço para correspondência: thatacristhina@hotmail.com todos os âmbitos da vida desse paciente sejam assistidos (Gomes \& Othero 2016).

Diante desse contexto, esta revisão de literatura visa levantar os estudos desenvolvidos em relação ao manejo da espiritualidade como ferramenta nos cuidados paliativos de pacientes oncológicos, proporcionando a estes melhor qualidade de vida. Este levantamento é necessário para que os profissionais de psicologia tenham subsídio e embasamento teórico para atuar de forma holística diante desse paciente.

\section{Espiritualidade}

Por volta de três séculos, a palavra "espiritualidade" passou a ser mais utilizada na sociedade e no universo acadêmico (Souza \& Soares, 2005).

Panzini et al. (2007) afirmam que a espiritualidade, ao contrário da percepção de muitos, não tem o mesmo significado que religiosidade, pois, enquanto a religiosidade prevê uma doutrina e um modo de adaptação para aqueles que a seguem, a espiritualidade consiste no significado ao qual o indivíduo dá para sua vida, sua capacidade de transcender e a forma com a qual processa suas vivências, ou seja, o modo de vida do sujeito.

A religiosidade é um sistema ao qual o indivíduo segue para buscar compreender sua relação com o divino e o sobrenatural, em que pauta suas escolhas nas doutrinas e preceitos que cada linha religiosa prediz, e é o canal que ele utiliza para entrar em contato com a espiritualidade (Arrieira et al., 2017).

Fornazari e Ferreira (2010) afirmam que a espiritualidade e a religiosidade são redutoras de ansiedade 
e medo para o indivíduo e, para este, assegurar-se de que suas ações sejam aprovadas por um ser supremo que prediz o que está certo ou errado e, em um segundo momento, beneficia aquele que segue o que lhe foi doutrinado, traz maior segurança e confiança em tomar decisões mediante conflitos.

Conforme Skinner (1998), a religião para o indivíduo é como uma sucursal de controle que advém da "ligação" deste com o supremo/ sobrenatural, de maneira a criar ou modificar comportamentos. Dessa maneira, a religião regula ao indivíduo por meio de punições ou reforços, suas escolhas e embasamentos para estas. $\mathrm{O}$ autor postula que será de acordo com as contingências de toda a vida do sujeito que ele estará mais próximo ou mais distante da espiritualidade/religiosidade, para elaborar as situações que se apresentam durante toda sua vivência.

É notável que a espiritualidade e a religiosidade são grandes colaboradoras para os enfermos, ainda mais para aqueles com diagnóstico de neoplasias. Evangelista et al. (2016) afirmam que elas podem ser aliadas do paciente na adaptação ao contexto de acometido por uma patologia e o estresse advindo dessa situação. Cada vez mais a espiritualidade vem sendo notada no contexto de assistência à saúde, e suas colaborações vêm sendo avaliadas (Fornazari \& Ferreira, 2010).

Durante anos, a espiritualidade e a ciência eram contrapostas, ocorrendo até conflitos entre ambas. Entretanto, agora, os resultados trazidos da junção de ambas vêm sendo cada vez mais estudados e apropriados pelos profissionais da saúde (Teixeira, Muller, \& Silva, 2004).

Jarros, Dias, Müller e Souza (2008) elucidam que a primeira "interface" entre espiritualidade e ciência surgiu no âmbito médico por meio das respostas conclusivas de exames de imagens que comprovaram mudanças e excitação cerebral em pacientes quando estão em contato com o espiritual. O autor afirma que, no lugar do distanciamento entre a ciência e a espiritualidade, surge um interesse recíproco pelo reconhecimento dos possíveis benefícios.

Atualmente, diversas pesquisas vêm comprovando que a espiritualidade melhora a qualidade de vida do indivíduo. Por exemplo, Elmescany e Barros (2015) apontaram que a espiritualidade é integrante do olhar terapêutico nos cuidados paliativos e que seu manejo como ferramenta de trabalho pela equipe de saúde produz grandes resultados, pois possibilita ao indivíduo enfermo formas diferentes de tratar as dificuldades advindas da patologia.

Teixeira et al. (2004) aprofundaram estudos e verificaram que indivíduos em tratamentos psicológicos que estão "conectados" com sua espiritualidade têm respostas melhores ao tratamento e diminuição de sintomas. Sua pesquisa indicou que aqueles com maior "ligação" com a espiritualidade são mais beneficiados com os tratamentos do que outros.

De acordo Panzini et al. (2007), o conceito ainda recente de qualidade de vida, que não é apenas a ausência de doenças, mas o bem-estar biopsiquicossocioespiritual do sujeito, tem grande valor para os profissionais da área da saúde e, também, está sendo reconhecido pela sociedade.

A qualidade de vida surgiu como um complemento para a medicina e demais áreas da saúde, pois assiste de forma abrangente o paciente, trabalhando a respeito da percepção das bases importantes da vida de uma pessoa. Por essa razão, a qualidade de vida se baseia na cultura, porque cada cultura tende a priorizar determinados aspectos (Teixeira et al., 2004).

A espiritualidade indiretamente produz qualidade de vida ao sujeito, pois é natural que aqueles que embasam sua vida em significações envoltas de um supremo se abstenham de comportamentos que julgue como errados mediante a visão desse ser superior. Assim, esse sujeito se afasta de comportamentos que são de risco para sua saúde física e mental, como uso de drogas lícitas e ilícitas (Oliveira \& Junges, 2012).

Oliveira e Junges (2012) explicam que questões de sofrimento psíquico são menos recorrentes em indivíduos espiritualmente direcionados, pois o grau de satisfação com sua vida e conquistas são maiores, além de terem maior tendência a ter boa qualidade de relacionamento sociofamiliar e até autoestima.

De acordo Panzini et al. (2007), a espiritualidade como umas das dimensões da qualidade de vida tem sido estudada mais a fundo, pelas mudanças significativas que pode causar para o sujeito.

Jarros et al. (2008) afirmam que o manejo da espiritualidade facilita a equipe multidisciplinar responsável pelo paciente, principalmente o psicólogo, na compreensão das orientações cognitivas que a espiritualidade tem sobre o indivíduo, na avaliação da sua condição de vida, seja no momento ou até antes do diagnóstico e finitude de tratamentos. Ainda, em um contexto devastador para o paciente e sua família, a espiritualidade pode ser preventiva aos transtornos mentais.

Entre as alternativas para melhora da qualidade de vida do sujeito em cuidados paliativos, os profissionais estão adicionando a espiritualidade como variável significativa que tem se mostrado como coadjuvante nesse aspecto da vida do indivíduo (Souza \& Soares, 2005).

Oliveira e Junges (2012) verificaram a ligação direta entre espiritualidade e qualidade de vida e relatam que, para que o indivíduo tenha qualidade de vida, ele precisa estar saudável mentalmente e ter equilíbrio em todas as esferas, o que é facilmente alcançado e estabelecido quando este indivíduo está espiritualmente amparado.

\section{Doença oncológica}

O termo "oncologia" se refere à ciência que estuda como se forma o câncer, como ele se instala e progride. A necessidade de uma área única e totalmente voltada a essa patologia surgiu por ser uma das maiores causas de óbito no Brasil e no mundo. Atualmente, o câncer é responsável por mais de seis milhões de mortes por ano, 
o que representa $12 \%$ das causas de morte no mundo (Guerra, Gallo, \& Mendonça, 2005).

Na perspectiva social, o câncer é a enfermidade mais associada à morte pelos indivíduos, em todas as regiões do mundo, até mesmo naquelas com diversas patologias recorrentes, sendo a segunda enfermidade que mais leva a óbito (Borges et al., 2006). Borges et al. (2006) completam que essa patologia se dá por razões multifatoriais, seja predisposição genética, exposição a fatores ambientais de risco, infecção por vírus, cigarro, entre muitos outros. Os autores complementam afirmando que, biologicamente, o câncer é explicado pela divisão excessiva e anormalidade das células.

Carvalho (2002) acredita que fatores psicológicos também possam corroborar para o avanço dessa patologia e piora na resposta aos tratamentos. Sua pesquisa levantou que dentre os aspectos apresentados pelos pacientes oncológicos, se encontra a problemática intrapsíquica.

Carvalho (2002) afirma que essa problemática é composta por depressão, medo, raiva, mudanças de humor; problemas sociais, como isolamento, estigma, mudança de papéis; e, por fim, os problemas da própria doença, como o próprio tratamento e mutilações. Com isso, o autor comprova que, sem o cuidado da dimensão psicológica do paciente oncológico, este não terá suporte suficiente para lidar com esses problemas.

Elias (2003) afirma que o profissional responsável pelo cuidado e tratamento do paciente se dedica a encontrar a melhor forma para cada apresentação da patologia e a melhor prescrição de tratamento, combinando-as para melhores resultados e até a cura da doença. Em muitos casos, nenhum tratamento é eficaz e o paciente passa a ter que lidar com a perspectiva de morte. Diante dessa situação, só o que se pode oferecer ao paciente são os cuidados paliativos (Evangelista et al., 2016).

\section{Paciente oncológico}

De acordo Geronasso e Coelho (2012), o paciente portador do diagnóstico de câncer, passa por um emaranhado de emoções permeadas por angústia, revolta, tristeza, medo entre outros aspectos, e esse misto de sentimentos pode gerar depressão e/ou ansiedade. As autoras ainda afirmam que os sintomas depressivos desse paciente são fruto da culpa que ele sente, com sentimentos de inferioridade, entre outros, enquanto a ansiedade é desenvolvida pelo pensamento futuro de como a doença vai progredir, os possíveis tratamentos e as perdas.

Além do paciente, Geronasso e Coelho (2012) comentam acerca da família e rede de apoio, que também sofrem prejuízos diretos com o diagnóstico, porque este traz a necessidade de reelaboração de papéis dentro desses contextos e novas táticas de vivência para lidar com as perdas e os caminhos que perpassarão após o diagnóstico.

Fornazari e Ferreira (2010) elucidam que, para lidar com o novo contexto, o paciente usa estratégias de enfrentamento, que são esforços cognitivos e/ou comportamentais direcionados para suprir a nova realidade imposta ao sujeito após diagnóstico. Esta realidade traz mudanças e demandas internas e externas, que geram sobrecarga aos recursos pessoais do paciente.

Benites Neme e Santos (2017) completam que tais estratégias, que serão descritas adiante, podem ser classificadas como focadas na problemática ou na resposta emocional causada pelo diagnóstico. Sendo focadas na problemática, os autores afirmam que o paciente tende a se "comportar" de forma ativa, empenhando-se em conhecer a patologia, seu funcionamento e seus efeitos, no sentido de resolução de problemas. Já as estratégias baseadas na resposta emocional se direcionam para uma maneira de compreensão dessas novas emoções geradas, para equilíbrio das respostas causadas pela descoberta da patologia. Entre os comportamentos advindos dessa estratégia, podem ser encontradas a negação e a esquiva (Benites et al., 2017).

Culturalmente, o câncer é a patologia que mais causa temor à sociedade, devido a tratamentos invasivos, hipótese de reincidência e, em muitos casos, o óbito. Esta também é uma das razões pelas quais o paciente, ao receber esse diagnóstico, depara com tamanho desespero, medo e se sente sem acolhimento (Guerra et al., 2005).

Mesmo com todos os avanços em tratamentos, medicamentos e os muitos resultados de cura que vêm crescendo pelo mundo, em muitos casos, a doença se torna insubmissa ao tratamento, e o paciente passa a ter de lidar com a perspectiva de morte, restando ao ele apenas os cuidados paliativos (Borges et al., 2006).

\section{Cuidados paliativos}

Em 2002, a Organização Mundial da Saúde (OMS) definiu, em diversos países e idiomas, os conceitos e princípios dos cuidados paliativos, além de sua importância, recomendando-os aos profissionais como prática necessária para com os acometidos de doenças incuráveis (Organização Mundial da Saúde, 1990).

Entre esses conceitos, encontram-se a noção de elucidação da morte por parte do paciente como processo natural da vida; a tópica de que os cuidados paliativos não adiantam ou afastam a morte, mas são ações focadas na melhoria da qualidade de vida dos pacientes e sua rede de apoio (Elmescany \& Barros, 2015).

De acordo com Melo, Valero e Menezes (2013), a atuação dessa equipe interdisciplinar consiste em melhorar a qualidade de vida do paciente terminal, oferecer-lhe condição para viver o mais ativamente possível até sua morte, abordar, em equipe, ações que atendam à necessidade do paciente e de sua família, auxiliar a família e a rede de apoio a lidarem com a patologia, o novo contexto e, também, com o luto.

Segundo Gomes e Othero (2016), cuidados paliativos são práticas relacionadas à preocupação integral com o sujeito, e é justamente o olhar global que diferencia 
esse cuidado dos demais com intenção curativa. Além disso, as autoras completam afirmando que os cuidados paliativos buscam lidar com alternativas para melhora da qualidade de vida do paciente, por meio da prevenção de sintomas e do auxílio no enfrentamento da doença.

Segundo Melo et al. (2013), os cuidados paliativos também implicam em reconhecer o sujeito como parte de seu tratamento, dando-lhe autonomia em participar das medidas que vão ser tomadas para aprimorar sua qualidade de vida. O exemplo trazido pelas autoras é que, em muitos casos, o indivíduo escolhe receber o tratamento em casa para poder ter seus últimos dias em um ambiente aconchegante e rodeado por sua família.

\section{Psicologia e cuidados paliativos}

Carvalho (2002) aponta que uma das subespecialidades da oncologia é a psiconcologia. Por ser uma doença de impacto total na vida do indivíduo, o câncer faz que seja necessária uma equipe multidisciplinar para seu tratamento e acompanhamento. E a psicologia é uma integrante que compõe uma dessas equipes.

De acordo com Pereira e Branco (2016), o psicólogo voltado para a área oncológica direciona sua atuação a compreender o impacto da doença no funcionamento emocional do indivíduo e de sua rede, verificando a influência das variáveis psicológicas e comportamentais do convívio com a nova realidade para o paciente. As autoras afirmam que esses profissionais dão apoio psicossocial e psicoterápico para o paciente e suas famílias, e esse cuidado proporciona auxílio para uma melhor forma de enfrentamento do câncer e dos impactos gerados por ele, facilitando a compreensão de modos mais saudáveis que melhorem a qualidade de vida do doente.

Para Arrieira et al. (2018), fazem parte das funções do profissional da psicologia na equipe de cuidados paliativos a atuação promovendo a humanização da equipe para tratar o paciente e sua família, a compreensão dos fenômenos psíquicos desenvolvidos pelo paciente diante da perspectiva de morte, entendimento do funcionamento das relações do paciente, suporte e orientação à família e à equipe de cuidado.

Dentre as intervenções, encontram-se a avaliação do diagnóstico do paciente e da história clínica, das relações familiares e dos papéis desenvolvidos dentro desse contexto, transmitir os resultados as conclusões sobre os caminhos de tratamento para equipe, trabalhar com o paciente seus estilos de enfrentamento, promover a aproximação do paciente com o meio social e sua família, além de trabalhar a elaboração do paciente diante dessa nova perspectiva de vida (Melo et al., 2013).

Nesse novo modelo de cuidado e atenção - os cuidados paliativos -, o psicólogo também tem sua participação ativa, com uma perspectiva de integralidade ao olhar o sujeito de cuidado. Sua atuação se aplica não só ao paciente, mas também a família, buscando diminuir significativamente a dor do paciente, seja de ordem física ou psicológica (Ferreira, Lopes, \& Melo, 2011).

Segundo Evangelista et al. (2016), os cuidados paliativos, quando direcionados a pacientes terminais, têm a psicoterapia como ferramenta de suma importância no sentido de auxiliar o enfrentamento da condição imutável de morte.

Melo et al. (2013) afirmam que os instrumentos utilizados como ferramenta para o profissional da psicologia são embasados em avaliações para compreender o funcionamento psíquico do paciente após diagnóstico. Dentre esses instrumentos estão a escala de avaliação psicossocial, que levanta, por meio das respostas do paciente, os fatores de risco e comportamentais que são indicadores do impacto emocional causado pelo diagnóstico. Os autores também citam a entrevista semiestruturada, que indica não só as respostas verbais do paciente, mas também as não verbais, comoreações físicas e respostas corporais (Pereira \& Branco, 2016).

Segundo Jarros et al. (2008), o profissional psicólogo inserido na equipe multidisciplinar em cuidados paliativos do paciente oncológico pode utilizar a espiritualidade como ferramenta auxiliadora de sua prática. $\mathrm{O}$ autor afirma que a espiritualidade, uma das dimensões que compõe a qualidade de vida do sujeito, pode ser aquela que preconiza maior adaptação do paciente à situação de finitude da vida e todos os impactos advindos da doença oncológica. As autoras ainda afirmam que o manejo da espiritualidade como ferramenta pode ser aplicado para aproximação e criação de vínculo com o paciente e sua família, além de transmitir a estes que o intuito do atendimento e do cuidado é tornar a vida do paciente o mais confortável possível, e que todas as demandas e necessidades trazidas por ele será assistida e enquadrada no plano de auxílio.

Pinto (2009) evidencia que a espiritualidade, sendo a expressão da religiosidade, pode ser utilizada pelo profissional psicólogo para facilitar ao paciente em cuidados paliativos a ressignificação de sua nova realidade e, também, na escolha de intervenções a serem realizadas no plano de finalização de assuntos que, pela visão do paciente, não podem ser deixados como inacabados. Portanto o estudo da espiritualidade nos cuidados paliativos oncológicos se mostra necessário como uma variável exponencial e coadjuvante no tratamento multidisciplinar.

\section{Metodologia}

Para atingir os objetivos propostos neste trabalho, utilizou-se o método de revisão bibliográfica, que permite o levantamento de publicações coerentes em determinada área de conhecimento, possibilitando, neste caso, o alcance de conhecimentos científicos produzidos em oncologia, psiconcologia, cuidados paliativos, espiritualidade e a correlação entre essas esferas (Echer, 2001). Este trabalho foi elaborado a partir de uma revisão de literatura feita nas bases de dados on-line PePSIC, SciELO e Lilacs, 
tendo como critério trabalhos produzidos no período de 1990 a 2020 nas bases de estudos nacionais.

Os unitermos de pesquisa utilizados foram: "espiritualidade", "cuidados paliativos", "qualidade de vida", "psiconcologia" e seus sinônimos. Como intersecção de termos, foram feitas as junções: "espiritualidade x qualidade de vida", "espiritualidade x cuidados paliativos", "espiritualidade x doença oncológica", "espiritualidade x psicologia", "doença oncológica x psicologia", "cuidados paliativos x psicologia".

Os critérios de inclusão foram artigos que tratassem da temática da espiritualidade e de sua contribuição sendo utilizada como ferramenta nos cuidados paliativos, tendo em vista também compreender a psicologia como área integradora entre os conteúdos de espiritualidade e cuidados paliativos.

Os critérios de exclusão estão relacionados a publicações anteriores ao ano de 1990, aquelas que tinham a religiosidade/espiritualidade apenas com embasamento místico, e não científico, publicações em que o enfoque era no tratamento do paciente oncológico sem a perspectiva de não resultado, cuidados paliativos e espiritualidade relacionados a outras patologias e, por fim, aqueles que restringiam a doença oncológica e os cuidados paliativos em acometimento específico da patologia.

Foi encontrado, nas três bases de dados, o total de 690, sendo 71 da base de dados SciELO, 54 da PePSIC e 565 da Lilacs.

Por meio dos critérios de inclusão e exclusão, realizou-se a análise dos artigos, dentre os quais 661 foram excluídos e 29 foram selecionados como objeto de estudo, lidos e resumidos na íntegra.

Foram excluídos os artigos que, embora tratassem da temática, tivessem unitermo específico, não se correlacionavam com os objetivos desta revisão, trabalhos que não se enquadravam no período tido como critério para levantamento, artigos que tratavam de outra patologia e aqueles que não eram de origem nacional.

Os artigos e estudos selecionados correspondiam aos objetivos deste trabalho e colaboravam para a explicação da correlação dos objetivos de pesquisa, além de agregarem subsídio para a fundamentação teórica desta revisão bibliográfica.

\section{Resultados e discussão}

Por meio da revisão de literatura, seleção e exclusão dos artigos advindos das bases de dados que correspondiam ou não aos critérios de inclusão, identificaram-se diversos resultados acerca dos objetivos desta revisão, ou seja, resultados que mostram o papel da espiritualidade nos cuidados paliativos do paciente oncológico.

Notou-se que esses resultados podem ser divididos entre os grupos de indivíduos que perpassam, acompanham, realizam e fazem parte do ambiente oncológico e dos cuidados paliativos. Estes grupos são os próprios pacientes, a equipe multidisciplinar responsável pelo cuidado e os familiares e a rede de apoio dos pacientes. Nesta revisão, a atuação do profissional psicólogo será vista separadamente, já que este é um dos objetos desta pesquisa.

\section{Grupo 1:paciente oncológico}

Em todo o contexto dos cuidados paliativos, do manejo da espiritualidade e da aceitação da morte como processo inerente a vida, o paciente paliativo é o centro. Observou-se que, para cada faixa etária, a espiritualidade se apresentou de uma maneira diferente e que, quanto mais idade tiver o paciente, mais ele utilizará essa ferramenta para lidar com seu processo. Elias (2003) corroborou esta afirmação ao constatar em sua pesquisa que, comparando-se crianças e adolescentes, estes apresentam dor psíquica e espiritual, enquanto aquelas, apenas dor espiritual. Devido à concepção social de desenvolvimento humano, a morte é pouco tratada e aceita em idade infantil e adolescente (Bee, 1997). Pode-se conjecturar que, por esta razão, haja uma limitação nos achados deste estudo na concepção dos cuidados paliativos para os pacientes dessa idade e, também, para seus cuidadores e sua rede.

Esta pesquisa evidenciou o trabalho de Palline, Ottati, Cremaso e Cunha (2019), que constataram em seu estudo que todos os pacientes por eles investigados falaram sobre os benefícios da espiritualidade, o que corrobora com os resultados obtidos por Menezes, Kameo, Valença e Moco (2018), ao identificarem, em seu estudo com 42 pacientes oncológicos, que o uso da espiritualidade para enfrentamento da patologia é positivo.

Como resultado desta revisão, encontrou-se que a espiritualidade mostra-se como um benefício ao paciente oncológico para compreender sua situação. Fornazari e Ferreira (2011) identificaram em seu estudo com pacientes oncológicos que estes, mesmo tendo uma linha espiritual e seguimento religioso antes do diagnóstico, relataram maior procura pela religião e ajuntamentos religiosos depois de diagnosticados.

Este trabalho ainda verificou, na pesquisa de Benites et al. (2017), que, diante da perspectiva de morte, o paciente volta-se à busca pelo sentido de vida. Esta afirmação completa-se com as reflexões de Freitas, Vieira, Guerra e Pasini (2016), que, em sua pesquisa com 30 pacientes oncológicos, identificaram o conceito de fé e crença espiritual mais expressiva após diagnóstico em seus pacientes.

Diante dos achados supracitados, é possível corroborar os presentes dados com os achados de Erickson (1976), que, ao trabalhar em sua teoria sobre as fases do desenvolvimento, pontua que o indivíduo em idade avançada (final dos 60 anos adiante) irá deparar com duas questões: a integralidade versus a desesperança. Neste contexto, Bee (1997) descreve que, perante o diagnóstico de morte, o sujeito que alcançou a integralidade terá maior facilidade em aceitá-la como processo natural da vida, pois olhará sua vivência com satisfação e alegria. 
Torna-se inerente a este resultado a espiritualidade como auxiliadora no processo de integralidade, pois possibilita ao paciente ressignificar suas experiências.

Para corroborar com os achados supracitados, também se ratifica que a espiritualidade produz ao paciente, em condição final, maior qualidade de vida, o que integra os objetivos dos cuidados paliativos, como pontuam Teixeira et al. (2004) em seus estudos. Além disso, por meio do manejo da espiritualidade, os pacientes demonstram maior paz e tranquilidade ao tratar da finitude da vida (Evangelista et al., 2016).

Em relação à busca pela compreensão do acometimento da patologia e, em seguida, da impossibilidade de cura-e diante do processo de morrer-, evidenciou-se nesta pesquisa que a espiritualidade auxilia na compreensão do sentido da vida e na finalização dos processos abandonados durante a vivência para o paciente (Freitas et al., 2016). Este dado pode ser corroborado com o desenvolvimento humano quando Bee (1997) descreve que será saudável aos adultos mais velhos, trazerem ao contexto de finalização da vida o conceito de reminiscência para se lembrarem da vida de forma positiva.

Este estudo verificou, na revisão integrativa realizada por Silva (2011), que a espiritualidade atua como papel reconfortante ao paciente paliativo. De acordo o levantamento realizado nesta revisão de literatura, as publicações referentes ao tema aumentaram significativamente a partir de 2014, por exemplo, a pesquisa realizada por Evangelista et al. (2016), que, tendo o mesmo objetivo deste estudo, encontrou 39 publicações referentes a esta temática.

\section{Grupos 2: profissionais da equipe multidisciplinar}

Em relação a este grupo, os profissionais da equipe multidisciplinar responsável pelos cuidados paliativos desses pacientes, os resultados direcionados à relação entre a espiritualidade e estes profissionais estão atrelados ao manejo da espiritualidade como ferramenta para maior compreensão do impacto do diagnóstico ao paciente e sua rede. No que tange a este tópico, esta revisão identificou o trabalho de Peres, Arantes, Lessa e Caous (2007), que verificaram que a espiritualidade é utilizada pelos profissionais como ferramenta de cuidado ao paciente paliativo.

Embora a temática espiritual não faça parte de todas as técnicas aplicadas por esses profissionais, os artigos selecionados evidenciaram que a espiritualidade é um assunto recorrente nos atendimentos. Além disso, também foi possível observar que, para os profissionais, o cuidado da dimensão espiritual do paciente aumenta e corrobora para sua qualidade de vida. Um estudo realizado por Oliveira, Buss, Rutz, Coelho e Habekost (2016) verificou, acerca das reflexões de uma equipe de cuidados paliativos, que, para estes profissionais, há correlação entre a espiritualidade e os cuidados paliativos. Observou-se, na pesquisa de Gobatto e Araujo (2013), que mesmo os profissionais não religiosos praticantes têm contato com a temática da espiritualidade para cuidado dos pacientes, ouvindo sobre sua religião e se interessando em apreender. Em sua tese, Espíndula, Valle e Belo (2010) corroborando este resultado, selecionaram profissionais de áreas distintas e pertencentes à equipe de cuidados paliativos, solicitando a estes que utilizassem a técnica relaxamento, imagens mentais, espiritualidade (Rime) e, depois, descrevessem suas concepções. Todos os profissionais, até os que se mantinham teoricamente amparados na separação entre espiritualidade e ciência, relataram sentir resultados positivos em seus pacientes com o manejo da técnica.

\section{Grupo 3: família e rede do paciente}

Esta revisão também verificou que os resultados do levantamento nas bases de dados, em relação à família e rede do paciente paliativo, mostraram o contexto da espiritualidade. Esses familiares demonstram que a espiritualidade age para conforto e auxílio na elaboração do luto futuro e da despedida. Exemplo disso é o trabalho de Barbosa, Ferreira e Costa (2017), que comprovou que, mesmo de maneiras diferentes, a espiritualidade será utilizada como estratégia de enfrentamento para o familiar do paciente terminal.

Além disso, os resultados denotam também que a relação da espiritualidade para o familiar que se voluntaria a cuidar de forma intensiva do paciente oncológico se assemelha à da equipe multidisciplinar, no sentido de gerar reflexões acerca do sentido da vida, qualidade das relações e apoio em crenças, é o que comprova os estudos de Farinhas, Wedling e Zanon (2013). Corroborando os resultados encontrados nesta revisão, Bee (1997) aponta que os familiares que participam do cuidado do paciente terminal se sentem mais satisfeitos e relatam melhor compreensão em relação à inevitabilidade da morte, se comparados aos familiares que apenas acompanham o cuidado hospitalar.

Além disso, Guimarães e Lipp (2011) identificaram que, para o familiar cuidador, a sobrecarga é física e psíquica, pois, enquanto física, ele se vê responsável a proporcionar para o paciente $\mathrm{o}$ atendimento de qualquer dificuldade que este verbalize e conciliar com seus outros afazeres enquanto sujeito, e também as quais ele enxerga como necessidade. Já enquanto psíquica, ele lida com o luto iminente que trará a perda e o suporte para a dor psíquica desse paciente.

\section{Grupo 4: profissional da psicologia}

Este estudo identificou também que o psicólogo será, na equipe multiprofissional, o profissional que mais tem ferramentas para acolhimento do paciente e de sua rede, além de ser o que tem maior compreensão 
da espiritualidade como auxiliadora no processo, como afirmam Domingues et al. (2013).

O trabalho de Elmescany e Barros (2015) também verificou que a terapia ocupacional, para o paciente paliativo em fase terminal, o auxilia a viver com sentido. Complementando esta afirmação sobre o cuidar, os estudos de Rezende, Gomes e Machado (2014) apresentaram que, na assistência psicológica dos cuidados paliativos, o auxílio integral ao paciente deve ter prioridade sobre o cuidar.

Os resultados das pesquisas de Porto e Lustosa (2010) apontaram que o profissional psicólogo é essencial na equipe de cuidados paliativos, pois este é aquele que vai compreender os impactos psíquicos ao paciente e facilitar sua maior conexão com as dimensões de sua vida para elaborar a nova condição, ou seja, aproximá-lo da espiritualidade.

Embora Gobatto e Araújo (2010) tenham verificado que tornou-se papel do profissional compreender e avaliar o uso do coping religioso-espiritual em situações de finitude de vida, Ferreira et al. (2011) apontam que há escassez de publicações acerca da atuação do psicólogo como integrante da equipe de cuidados paliativos. Nesse sentido, verifica-se que esse pode ser um fator que dificulta a este profissional realizar propostas de intervenção.

Por fim, notou-se, por meio da revisão de literatura, que o tema da espiritualidade associada aos processos de qualidade de vida e integrante para o bem estar do sujeito é ainda muito recente em bases bibliográficas, e que os estudos para gerar subsídio ao seu manejo, para os profissionais da saúde, ainda estão em desenvolvimento. De acordo os estudos de Ferreira et al. (2011), ainda existe a necessidade de aprofundamento de estudos científicos sobre a temática.

\section{Limitação do estudo}

Acerca das limitações encontradas na produção desta pesquisa, verificou-se restrição em resultados sobre a "interface" dos cuidados paliativos para os pacientes terminais em idade infantil e adolescente, e, também, em relação ao impacto gerado ao familiar desse paciente.

\section{Conclusão}

As formas que a espiritualidade pode influenciar positivamente no tratamento oncológico e cuidados paliativos são: auxiliando o paciente oncológico a lidar de modo saudável com a concepção da morte, passando a compreendê-la como processo natural da vida; proporcionar ao paciente oncológico um enfrentamento saudável em relação ao novo contexto que irá perpassar, auxiliando-o a elaborar e ressignificar suas vivências e relações. Ao se tratar da equipe multidisciplinar de cuidados, a espiritualidade auxilia positivamente para que ela compreenda o impacto total causado ao paciente pelo diagnóstico e realize intervenções no contexto biopsicossocioespiritual para cuidado do paciente e acolhimento de sua família.

O manejo da espiritualidade na qualidade de vida do paciente oncológico terminal se trata de compreender não só o acometimento físico da patologia e o impacto psicológico da perspectiva de morte, mas a concepção total das vivências do sujeito e processo de resiliência em seus últimos dias, além de proporcionar a este vivê-los com sentido.

Para além disso, verificou-se que a espiritualidade tem influência positiva nos cuidados paliativos de pacientes oncológicos e, colaborando para que estes sejam assistidos de forma integral, a espiritualidade também agrega subsídio à equipe multidisciplinar no tocante a tratar com os pacientes sobre a finitude da vida e o processo linear até a morte. A espiritualidade é utilizada com o paciente como ferramenta de enfrentamento diante da condição de morte e de auxílio para um sentido de vida em seus últimos dias.

Para os cuidados paliativos, é necessário que se abandone a concepção de atitude curativa e se priorize a noção de cuidado, e é a partir dessa ótica que esse tipo de cuidados possibilita qualidade de vida aos pacientes.

O paciente oncológico, entre as mais diversas patologias, é aquele que apresenta não só os acometimentos específicos da doença, que envolve excessiva dor e mutilações, mas também seus estigmas, que acompanham todo o processo curativo até o esgotamento dessas possibilidades.

É por meio do olhar integral que os CP propõem ao profissional que eles direcionem sua prática e assistência ao paciente paliativo. A sensação de cuidado total e a noção de compreensão sobre sua condição geral e de ser participante ativo dessa realidade confere ao paciente qualidade de vida em seus últimos dias.

O manejo da espiritualidade, então, se torna fundamental para proporcionar ao paciente os melhores cuidados, resultando em qualidade de vida e bem-estar geral, porque se trata não só de uma ferramenta ao profissional, para assistir completamente ao paciente, mas também para a família e para o próprio indivíduo, de instrumento de enfrentamento e auxílio na compreensão e direção após diagnóstico.

Em relação às limitações deste estudo, sugere-se que mais pesquisas sejam realizadas na área de interface entre os cuidados paliativos e os pacientes infantis/ adolescentes para que se compreenda a espiritualidade para este indivíduo e sua rede.

Se houver melhor educação sobre a temática apresentada nesta revisão, aumenta-se a possibilidade de ajudar o profissional de saúde em sua prestação de serviço, incluindo evitar ser distraído por seus preconceitos, medos e restrições. Além disso, possibilitar um melhor atendimento ao paciente e sua família (Best, Leget, Goodhead, \& Peal, 2020). 


\section{Spirituality in palliative care of oncological patients}

Abstract: The spirituality as a tool in palliative healthcare of oncology patients, has been increasingly studied and approached in the academic context, as an integrant of life quality improvement. The present study aimed to analyze how spirituality can positively influence oncology treatment and palliative care and contribute to the quality of life improvement of these patients. Through this literature review and the inclusion criteria, there had been 29 articles selected. Spirituality is used as a tool by the $\mathrm{CP}$ team as an aid to understanding de impact caused by the diagnosis and full-time patient care, it enables the psychologist professional to assist and intervene fully about the patient and its network; on the patient and his family, the spirituality acts as a coping strategy, safety, and comfort. The present study has found limitations around the study in children, adolescents, and their network.

Keywords: spirituality, palliative care, cancer patient, psychology.

\section{Spiritualité dans les soins palliatifs des patients oncologiques}

Resumé : La spiritualité en tant qu'outil de soins palliatifs des patients atteints de cancer est de plus en plus abordée dans le contexte académique, dans le cadre de la qualité de vie. La présente étude visait à analyser comment la spiritualité peut influencer positivement le traitement du cancer et les soins palliatifs et contribuer à améliorer la qualité de vie de ces patients. Grâce à cette revue de la littérature, 29 articles ont été sélectionnés. La spiritualité est utilisée comme outil par l'équipe de soins palliatifs pour aider à comprendre l'impact provoqué par le diagnostic et la prise en charge globale du patient, permettant au psychologue professionnel d'intervenir sur le patient et son réseau; pour le patient et sa famille, la spiritualité agit comme une stratégie d'adaptation et de confort. La présente étude a révélé des limites concernant la recherche avec des patients enfants / adolescents et leur famille.

Mots-clés : spiritualité, soins palliatifs, patient oncologique, psychologie.

\section{Espiritualidad en la atención paliativa del paciente oncológico}

Resumen: La espiritualidad como herramienta para los cuidados paliativos del paciente oncológico se ha abordado cada vez más en el contexto académico como parte de la calidad de vida. El presente estudio tuvo como objetivo analizar cómo la espiritualidad puede influir positivamente en el tratamiento del cáncer y los cuidados paliativos, así como contribuir a mejorar la calidad de vida de estos pacientes. Se seleccionaron 29 artículos brasileños que abordaron la espiritualidad como una variable positiva en el afrontamiento a los cuidados paliativos tanto para los pacientes como sus familias y el equipo multidisciplinario. La espiritualidad es utilizada por el equipo de cuidados paliativos como herramienta de ayuda en la comprensión del impacto que ocasiona el diagnóstico y la atención integral del paciente, permitiendo al psicólogo profesional intervenir sobre el paciente y su red. Para el paciente y su familia, la espiritualidad actúa como estrategia de afrontamiento y consuelo. El presente estudio encontró limitaciones con respecto a la investigación con pacientes niños/adolescentes y su familia.

Palabras clave: espiritualidad, cuidados paliativos, paciente oncológico, psicología.

\section{Referências}

Arrieira, I. C. O., Thofehrn, M. B., Porto, A. R., Moura, P. M. M., Martins, C. L., \& Jacondino, M. B. (2018). Espiritualidade nos cuidados paliativos: experiência vivida de uma equipe interdisciplinar. Revista da Escola de Enfermagem da USP, 52, e03312. doi: 10.1590/ s1980-220x2017007403312

Arrieira, I. C. O., Thoferhn, M. B., Schaefer, O. M., Fonseca, A. D., Kantorski, L. P., \& Cardoso, D. H. (2017). O sentido do cuidado espiritual na integralidade da atenção em cuidados paliativos. Revista Gaúcha de Enfermagem, 38(3), e58737. doi: 10.1590/19831447.2017.03.58737

Barbosa, R. M. M., Ferreira, J. L. P., Melo, M. C. B., \& Costa, J. M. (2017). A espiritualidade como estratégia de enfrentamento para familiares de pacientes adultos em cuidados paliativos. Revista da SBPH, 20(1), 165-182.

Bee, H. (1997). O ciclo vital. São Paulo, SP: Artmed.

Benites, A. C., Neme, C. M. B.o, \& Santos, M. A. (2017). Significados da espiritualidade para pacientes com câncer em cuidados paliativos. Estudos de Psicologia 
(Campinas), 34(2), 269-279. doi: 10.1590/198202752017000200008

Best, M., Leget, C., Goodhead, A., \& Peal, P. (2020). Um white paper do EAPC sobre educação multidisciplinar para cuidado espiritual em cuidados paliativos. $B M C$ Palliat Care, 19(9), 1-10. 10.1186/s12904-019-0508-4

Borges, A. D. V. S., Silva, E. F., Mazer, S. M., Toniollo, P. B., Valle, E. R. M., \& Santos, M. A. (2006). Percepção da morte pelo paciente oncológico ao longo do desenvolvimento. Psicologia em Estudo, 11(2), 361-369. doi:10.1590/S1413-73722006000200015

Carvalho, M. M. (2002). Psico-oncologia: história, características e desafios. Psicologia USP, 13 (1), 151-166. doi: 10.1590/ S0103-65642002000100008

Domingues, G. R., Alves, K. O., Carmo, P. H. S., Galvão, S. S., Teixeira, S. S., \& Baldoino, E. F.. (2013). A atuação do psicólogo no tratamento de pacientes terminais e seus familiares. Psicologia Hospitalar, 11(1), 02-24.

Echer, I. C. (2001). A revisão de literatura no trabalho cientifico. Revista Gaúcha de Enfermagem, 22(2), 5-20. Recuperado de https://bit.ly/3oLrc8S

Elias, A. C. A. (2003). Re-significação da dor simbólica da morte: relaxamento mental, imagens mentais e espiritualidade. Psicologia: Ciência e Profissão, 23(1), 92-97. doi: 10.1590/S1414-98932003000100013

Elmescany, É. N. M., \& Barros, M. L. P. (2015). Espiritualidade e terapia ocupacional: reflexões em cuidados paliativos. Revista do Nufen, 7(2), 1-24. Recuperado de https://bit.ly/3DriSPM

Erickson, E. H. (1976). Identidade, juventude e crise (2a ed.). Rio Janeiro, RJ: Zahar.

Espíndula, A. J., Valle, E. R., Belo, A. A. (2010). Religião e espiritualidade: um olhar de profissionais de saúde. Revista Latino Americana de Enfermagem, 18(6), 1-8.

Evangelista, C. B., Lopes, M. E. L., Costa, S. F. G., Batista, P. S. S., Batista, J. B. V., \& Oliveira, A. M. M. (2016). Cuidados paliativos e espiritualidade: revisão integrativa da literatura. Revista Brasileira de Enfermagem, 69(3), 591-601. doi: 10.1590/0034-7167.2016690324i

Farinhas, G. V., Wendling, M. I., \& Dellazzana-Zanon, L. L. (2013). Impacto psicológico do diagnóstico de câncer na família: um estudo de caso a partir da percepção do cuidador. Pensando Famílias, 17(2), 111-129.

Ferreira, A. P. Q., Lopes, L. Q. F., \& Melo, M. C. B. (2011). O papel do psicólogo na equipe de cuidados paliativos junto ao paciente com câncer. Revista da SBPH, 14(2), 85-98. Recuperado de

Fornazari, S. A., \& Ferreira, R. E. R. (2010). Religiosidade/ espiritualidade em pacientes oncológicos: qualidade de vida e saúde. Psicologia: Teoria e Pesquisa, 26(2), 265-272. doi: 10.1590/S0102-37722010000200008.

Freitas, E. O., Vieira, M. M. S., Guerra, G. M., Tsunemi, M. H., Pessini, L. (2016). A influência da espiritualidade na qualidade de vida do paciente oncológico: reflexão bioética. Nursing, 17(222), 1266-1270.

Geronasso, M. C. H., \& Coelho, D. (2012). A influência da espiritualidade/religiosidade na qualidade de vida das pessoas com câncer. Saúde e Meio Ambiente, 1(1), 1-15. Recuperado de https://bit.ly/2YBQwn0

Gobato, C. A., \& Araújo, T. C. C. F. A. (2013). Religiosidade e espiritualidade em oncologia: concepções de profissionais de saúde. Psicologia USP, 24(1), 11-34.

Gomes, A. L. Z., \& Othero, M. B. (2016). Cuidados paliativos. Estudos Avançados, 30(88), 155-166. doi: 10.1590/ s0103-40142016.30880011

Guerra, M. R., Gallo, C. V. M., Mendonça, G. A. S. (2005). Risco de câncer no Brasil: tendências e estudos epidemiológicos mais recentes. Revista Brasileira de Cancerologia, 51(3), 227-234.

Guimarães, C. A., \& Lipp, M. E. N. (2011). Um olhar sobre o cuidador de pacientes oncológicos recebendo cuidados paliativos. Psicologia: Teoria e Prática, 13(2), 50-62. Recuperado de https://bit.ly/3mEhDph

Jarros, R. B., Dias, H. Z. J., Müller, M. C., \& Sousa, P. L. R. (2008). Estudo bibliométrico da produção brasileira na interface da psicologia com espiritualidade-religiosidade. Psic: Revista da Vetor Editora, 9(2), 251-258. Recuperado de https://bit.ly/3aiKvOe

Menezes, R. R., Kameo, S. Y., Valença, T. S., Mocó, G. A. A., \& Santos, J. M. J. (2018). Qualidade de vida relacionada à saúde e espiritualidade em pessoas com câncer. Revista Brasileira de Cancerologia, 64(1) 9-17.

Melo, A. C., Valero, F. F., \& Menezes, M. (2013). A intervenção psicológica em cuidados paliativos. Psicologia, Saúde \& Doenças, 14(3), 452-469. Recuperado de https://bit.ly/3iGl6Cz

Oliveira, M. R., \& Junges, J. R. (2012). Saúde mental e espiritualidade/religiosidade: a visão de psicólogos. Estudos de Psicologia (Natal), 17(3), 469-476. doi: 10.1590/S1413-294X2012000300016

Organização Mundial da Saúde. (2002). Programas nacionais de controle do câncer: políticas e diretrizes gerenciais ( $2 \mathrm{a}$ ed.). Genebra: OMS.

Pallini, A. C., Ottati, F., Cremasco, G. S., \& Cunha, F. A. (2019). Percepções de pacientes oncológicos sobre espiritualidade: um estudo qualitativo. Psicologia para América Latina, (32), 169-179.

Panzini, R. G., Rocha, N. S., Bandeira, D. R., \& Fleck, M. P. A. (2007). Qualidade de vida e espiritualidade. Archives of Clinical Psychiatry (São Paulo), 34(Suppl. 1), 105-115. doi: 10.1590/S0101-60832007000700014

Pereira, T. B., \& Branco, V. L. R. (2016). As estratégias de coping na promoção à saúde mental de pacientes oncológicos:umarevisãobibliográfica.RevistaPsicologia e Saúde, 8(1), 24-31. doi: 10.20435/2177093X2016104

Peres, M. F. P, Arantes, A. C. L. Q., Lessa, P. S., Caous, C. A. (2007). A importância da integração da espiritualidade e da religiosidade no manejo da dor e dos cuidados paliativos. Archives of Clinical Psychiatry, 34(1), 82-87.

Pinto, É. B. (2009). Espiritualidade e religiosidade: articulações. Revista de Estudos da Religião, 4(1), 68-83. Recuperado de https://bit.ly/3DoK9Ch

Porto, G., \& Lustosa, M. A. (2010). Psicologia hospitalar e cuidados paliativos. Revista da SBPH, 13(1), 76-93. 
Rezende, L. C. S., Gomes, C. S., \& Machado, M. E. C. (2014). A finitude da vida e o papel do psicólogo: perspectivas em cuidados paliativos. Revista Psicologia e Saúde, 6(1) 28-36.

Silva, D. (2011). Significados e práticas da espiritualidade no contexto dos cuidados paliativos em pacientes oncológicos adultos. Clinical and Biomedical Research, 31(3), 353-358.

Skinner, B. F. (1998). Ciência e comportamento humano [Internet]. Recuperado de https://bit.ly/3j627Sk

Souza, J. C, \& Soares, A. S. (2005). Espiritualidade e qualidade de vida. Revista de Psiquiatria do Rio
Grande do Sul, 27(2), 218-219. doi: 10.1590/S010181082005000200013

Teixeira, E. F. B., Muller, M. C., Silva, J. D. T. (2004). Espiritualidade e qualidade de vida. Porto Alegre, RS: EDIPUCRS.
Recebido: 13/01/2021

Revisado: 06/09/2021

Aprovado: 27/09/2021 